\title{
BREAKDOWN OF A SHALLOW WATER EQUATION*
}

\section{H. P. MCKEAN ${ }^{\dagger}$}

1. Introduction. The 1-dimensional shallow water equation:

$$
\begin{aligned}
& \frac{\partial v}{\partial t}+v \frac{\partial v}{\partial x}+\frac{\partial p}{\partial x}=0 \quad \text { with "pressure" } \\
& p(x)=\frac{1}{2} \int_{-\infty}^{\infty} e^{-|x-y|}\left(v^{2}+\frac{1}{2} v^{\prime 2}\right) d y
\end{aligned}
$$

was noted by FOKAS-FUCHSTEINER [1981] as being formally integrable. CAMASSA-HOLM [1993] rediscovered it from a hydrodynamical point of view and developed it to a large degree, both there and in CAMASSA-HOLM-HYMAN [1994]. Their most striking discovery is that it has (peaked) solitons $v(t, x)=\frac{1}{2} p e^{-|x-q-p t / 2|}$ with constant $q$ and $p$, capable of being superposed in an elementary way, as sums $v(t, x)=\frac{1}{2} \sum_{i=1}^{n} p_{i}(t) e^{\left|x-q_{i}(t)\right|}$, where now the $q$ 's and $p$ 's obey the equations $q^{\bullet}=$ $\partial H / \partial p, p^{\bullet}=-\partial H / \partial q$ of the geodesic flow in the co-tangent bundle of $\mathbb{R}^{n}$, equipped with the inverse metric tensor $\frac{1}{2} e^{\left|q_{i}-q_{j}\right|}: 1 \leq i, j \leq n$, i.e., with Hamiltonian $H=\frac{1}{2}|p|^{2}+\frac{1}{4} \sum p_{i} p_{j} e^{-\left|q_{i}-q_{j}\right|}$. From this, it is only a little jump to the realization that the full shallow water flow in, e.g., $C_{\downarrow}^{\infty}(\mathbb{R})$ is just a transcription of the geodesic flow in the group $D(\mathbb{R})$ of (smooth proper) diffeomorphisms of the line, equipped with an analogous Riemannian geometry; see MISIOLEK [1998] for the more general case $v \in \mathbb{R}+C_{\downarrow}^{\infty}(\mathbb{R})$ with the attendant BOTT-VISASORO group, and compare ARNOLD-KHESIN [1998] for background and other illustrations of the general principle involved. Not much of that is needed here: it will suffice to observe a) if $v(t)$ obeys the shallow water flow in, e.g., $C^{\infty}(\mathbb{R})$ and if $Q(t, x)$ solves $Q^{\circ}=v(t, Q)$ with $Q_{0}(x) \equiv x$, then $Q$ is a diffeomorphism of the line ${ }^{1}$, imitating the identity near $x= \pm \infty$, and obeying the geodesic flow in $D(\mathbb{R})$, and b) the shallow water flow, expressed by means of $m=v-v^{\prime \prime}$ as in $\left.*\right) m^{\bullet}+(m D+D m) v=0$, has first integrals in the form $m(Q) Q^{\prime 2}(x) \equiv m_{0}(x) .^{2} *$ ) shows that $m(Q)$ retains the general shape of $m_{0}(x)$, independently of $t \geq 0$, illustrating the utility of the diffeomorphism for the study of the flow; indeed, it is the principal tool for the question posed here: When, and if so how, does the shallow water flow breakdown?

Now, unlike $\mathrm{KdV}$, breakdown is common enough as already noted in CAMASSAHOLM [1993]: for example, if $\int_{-\infty}^{\infty}\left(v^{2}+v^{\prime 2}\right) d x \equiv H<\infty$, this being a constant of motion, then, from 5) below in the form $\frac{d}{d t} v^{\prime}(Q) \leq \frac{1}{2} v^{2}(Q)-\frac{1}{2} v^{\prime 2}(Q)$, you see that $v_{0}^{\prime}\left(x_{0}\right)<-\sqrt{H}$ at any place $x_{0} \in \mathbb{R}$ implies $v_{0}^{\prime}\left(t, x_{0}\right) \downarrow-\infty$ at some finite time.

To fix ideas, let us suppose that either $v_{0} \in C^{\infty}(\mathbb{R})$ is of period 1 or else $v_{0} \in$ $\mathbb{R}+C_{\downarrow}^{\infty}(\mathbb{R})$, the latter being the most interesting case physically, and let us also simplify life by requiring that $m \equiv v_{0}-v_{0}^{\prime \prime}$ has only a finite number of (proper) changes of sign (per period). Then the solution exists in its function class for all time $0 \leq t<\infty$ only if the points $x_{-}$where $m_{0}\left(x_{-}\right)<0$ lie wholly to the left of the points

*Received December 8, 1998; accepted for publication February 5, 1999.

†CIMS, 251 Mercer Street, NYC, U.S.A. (mckean@cims.nyu.edu). The work presented here was performed at the Courant Institute of Mathematical Sciences with the partial support of the National Science Foundation under NSF Grant No. DMS-9112664, which is gratefully acknowledged.

${ }^{1} Q^{\prime \bullet}=v^{\prime}(t, Q) Q^{\prime}$ and $Q_{0}^{\prime} \equiv 1$ forces $Q^{\prime}(x)>0$.

$2 \frac{d}{d t} m(Q) Q^{\prime 2}=\left[m^{\bullet}(Q)+m^{\prime}(Q) Q^{\bullet}\right] Q^{\prime 2}+2 m Q^{\bullet} Q^{\prime \bullet}=0$, as you will check. 
$x_{+}$where $m_{0}\left(x_{+}\right)>0$ (which never happens in the periodic case unless $m_{0}$ is of one sign). Contrariwise, if $m_{0}$ is not of such a shape, then the solution breaks down at some time $T<\infty$ : in fact, as $t \uparrow T, v^{\prime}(t, Q) \downarrow-\infty$ at one or more of the (time independent) roots $x_{0}$ of $m(Q)=0$; simultaneously, $Q^{\prime}\left(t, x_{0}\right) \downarrow 0$, and more- $Q(t, x)$ flattens out in the largest interval $a \leq x \leq b$ containing $x_{0}$ in which $m_{0}(x) \equiv 0$. This is how the diffeomorphism comes to the "edge" of the group; in particular, the geodesic flow in $D(\mathbb{R})$ is incomplete. The existence of $v(t, x): t<T, x \in \mathbb{R}$ is not discussed here: That is covered by the methods of CONSTANTIN-ESCHER [1998] and CONSTANTIN-McKEAN [1998]. The present paper deals only with breakdown; it is a technical amplification of McKEAN [1999] which reviewed the whole connection with the diffeomorphism group and explained the breaking in a simple case.

Acknowledgment. It is a pleasure to thank A. Constantin for listening with much patience to rough version of the proof described below.

2. Preliminaries. Keep in mind the constants of motion $m(Q) Q^{\prime 2}=m_{0}(x)$ and the moral they convey: that the points $x_{0}$ where $m(Q)$ changes sign are fixed in time. Note also the duality: that if $v(t, x)$ is a solution, then so is $-v(t,-x)$. This will cut down the number of cases to be treated. Now come some little tricks which will be continually in use. They obtain up to the breaking time $T \leq \infty$.

$$
\begin{aligned}
& \frac{d}{d t} e^{Q}\left(v^{\prime}-v\right)(Q)=-\frac{1}{2} e^{Q}\left(v^{\prime}-v\right)^{2}(Q)-\frac{1}{2} \int_{-\infty}^{Q} e^{y}\left(v^{\prime}-v\right)^{2}(y) d y \\
& \frac{d}{d t} e^{-Q}\left(v^{\prime}+v\right)(Q)=-\frac{1}{2} e^{-Q}\left(v^{\prime}+v\right)^{2}(Q)-\frac{1}{2} \int_{Q}^{\infty} e^{-y}\left(v^{\prime}+v\right)^{2}(y) d y \\
& \frac{d}{d t} v^{\prime}(Q)=\frac{1}{2} v^{2}(Q)-\frac{1}{2} v^{\prime 2}(Q)-\frac{1}{4} \int_{-\infty}^{\infty} e^{-|Q(x)-y|}\left(v^{\prime} \pm v\right)^{2}(y) d y
\end{aligned}
$$

in which the ambiguous signature is that of $y-Q(x)$.

6) $\quad|v|$ is bounded, independently of time, by a fixed constant $C$.

7) $v^{\prime}$ is bounded above by $2 C+\max v_{0}^{\prime}$.

8) $\quad v^{\prime}$ is bounded below by $-C$ if breakdown does not take place.

Proof. $e^{Q}\left(v^{\prime}-v\right)=-\int_{-\infty}^{Q} e^{y} m(y) d y$. Now differentiate by $t$, using $Q^{\bullet}=v(Q)$ and $-m^{\bullet}=(m v)^{\prime}+\frac{1}{2}\left(v^{2}-v^{\prime 2}\right)^{\prime}$, and integrate by parts to obtain 3). 4) follows by duality. 5) is obtained by combining 3) and 4). 6) is plain if $v \in C_{\downarrow}^{\infty}(\mathbb{R})$ : then $H=\int_{-\infty}^{\infty}\left(v^{2}+v^{\prime 2}\right) d x$ is a constant of motion and $v^{2}=2 \int_{-\infty}^{x} v v^{\prime} \leq H$. The periodic case is similar with constant of motion $H=\int_{0}^{1}\left(v^{2}+v^{\prime 2}\right) d x$ and $v^{2} \leq 2 H$. Likewise, if $v=c+w \in \mathbb{R}+C_{\downarrow}^{\infty}(\mathbb{R})$, then $c$ and $H=\int_{-\infty}^{\infty}\left(w^{2}+w^{\prime 2}\right) d x$ are constants of motion and $|v| \leq|c|+\sqrt{H}$. 7) now follows from 3), 4), and 6): $e^{ \pm Q}\left(v^{\prime} \mp v\right)(Q)$ decreases with time and one of $e^{Q}$ are $e^{-Q}$ exceeds 1 , so $v^{\prime}(Q) \leq e^{\mp Q}\left(v_{0}^{\prime} \mp v_{0}\right)(Q) \pm v(Q)$, etc. 8)follows from 5 ) and 6 ) by the reasoning of sect. 1 :

$$
\frac{d}{d t} v^{\prime}(Q) \leq \frac{1}{2} v^{2}(Q)-\frac{1}{2} v^{\prime 2}(Q) \leq \frac{1}{2} C^{2}-\frac{1}{2} v^{\prime 2}(Q)
$$

drives $v^{\prime}(Q)$ down to $-\infty$ in finite time at any place where $v_{0}^{\prime}(x)$ falls below $-C$. $\square$

3. Proof of Breakdown. It is to be proved that breakdown takes place if there is any positive "mass" $m_{0}$ to the left of some negative mass. Let $x=0$ be chosen as in the figure so that $m_{0}(x)$ and so also $m(Q)$ is positive near $x_{-}<0$ and negative 


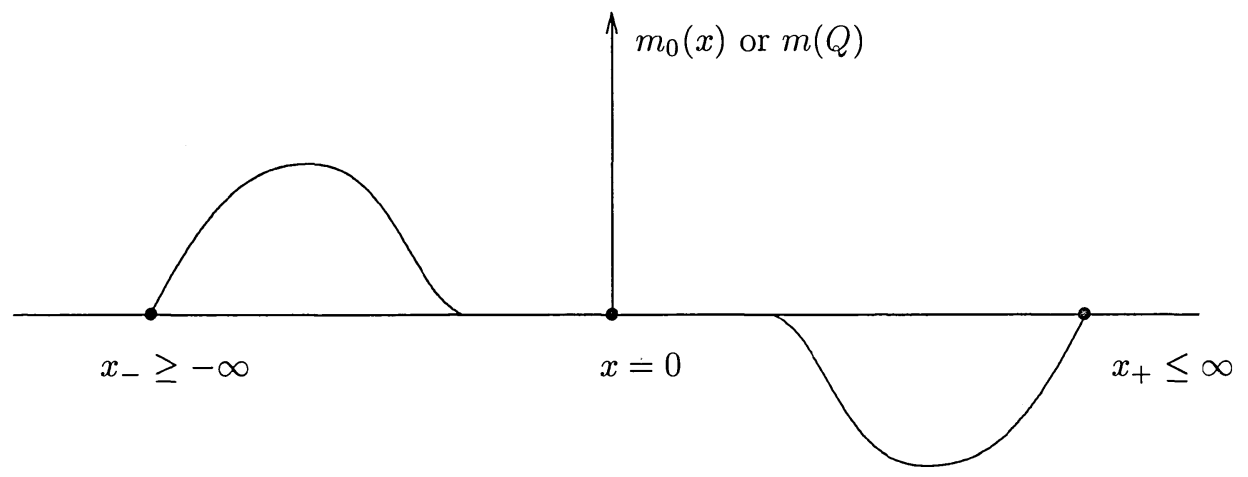

near $x_{+}>0$, a single interval containing $x=0$ being permitted for $m_{0}(x)$ to vanish. The picture is self-dual. Now if $\left(v^{\prime}-v\right)(Q)$ is ever negative at $x=0$, then 3$)$ in the form

$$
\frac{d}{d t} e^{Q}\left(v^{\prime}-v\right)(Q) \leq-\frac{1}{2}\left[e^{Q}\left(v^{\prime}-v\right)(Q)\right]^{2} e^{-Q}
$$

implies $\int_{0}^{\infty} e^{-Q} d t<\infty$ in the absence of breakdown; likewise, if $\left(v^{\prime}+v\right)(Q)$ is ever negative at $x=0$, then $\int_{0}^{\infty} e^{Q} d t<\infty$; and since $\int_{0}^{\infty} e^{ \pm Q} d t$ cannot both converge, so you can assume $\left(v^{\prime}-v\right)(Q) \geq 0$ at $x=0$ at all times $t \geq 0$, by duality. Now distinguish two cases according as $\left(v^{\prime}+v\right)(Q)$ is ever negative at $x=0$ or not.

Case 1: $\left(v^{\prime}+v\right)(Q) \geq 0$ at $x=0$ at all times $t \geq 0 .(d / d Q) e^{Q}\left(v^{\prime}-v\right)(Q)=$ $-e^{Q} m(Q)$ is $\leq 0$ between $x_{-}$and $x=0$, so $\left(v^{\prime}-v\right)(Q) \geq 0$ there, and $\left(v^{\prime}+\right.$ $v)(Q)$ likewise; in particular, $v^{\prime}(Q) \geq|v(Q)|$ for $x_{-} \leq x \leq 0$, and, in the absence of breakdown, so that $v^{\prime}(Q) \geq-C$ by 8$), 5$ ) implies

$$
+\infty>\int_{0}^{\infty} d t e^{-Q(x)} \int_{0}^{Q(x)} e^{y}\left(v^{\prime}-v\right)^{2}(y) d y \text { between } x=x_{-} \text {and } x=0
$$

Fix $x_{-}<a<c<b<0$ with $c=\frac{1}{2}(a+b)$ and $m_{0}(x)>0$ between $a$ and $b$. Then with $x=c$ in the last display,

$$
\begin{aligned}
+\infty & >\int_{0}^{\infty} d t e^{-Q(c)} \int_{Q(a)}^{Q(c)}\left[e^{y}\left(v^{\prime}-v\right)\right]^{2} e^{-y} d y \\
& >\int_{0}^{\infty} d t e^{-Q(c)}\left[e^{Q}\left(v^{\prime}-v\right) \text { at } x=c\right]^{2} \int_{Q(a)}^{Q(c)} e^{-y} d y \\
& =\int_{0}^{\infty} d t\left[\left(v^{\prime}-v\right)(Q) \text { at } x=c\right]^{2} \times\left[e^{Q(c)-Q(a)}-1\right] .
\end{aligned}
$$

This contradicts the fact that $m_{0}(x)>0$ for $a \leq x \leq b$, as will be seen in a few easy steps. 
STEP 1 uses $\left(v^{\prime}-v\right)(Q) \geq 0$ to obtain the simple bound:

$$
\begin{aligned}
{\left[\int_{c}^{b} \sqrt{m_{0}(x)} d x\right]^{2} } & =\left[\int_{c}^{b} \sqrt{m(Q)} d Q\right]^{2} \\
& \leq \int_{c}^{b} m(Q) d Q \times[\Delta Q \equiv Q(b)-Q(c)] \\
& =\int_{c}^{b}\left(v-v^{\prime}+v^{\prime}-v^{\prime \prime}\right)(Q) d Q \times \Delta Q \\
& \leq\left[\left(v^{\prime}-v\right)(Q) \text { at } x=c\right] \times \Delta Q
\end{aligned}
$$

STEP 2. Now let $v(Q)$ be negative at $x=b$. Then $v^{\prime}(Q) \geq|v(Q)|$ implies $v(Q) \leq 0$ down to $x=a$, so from $Q^{\bullet}=v(Q)$ and $Q^{\bullet \bullet}=v^{\prime}(Q) Q^{\prime}$, it appears that

$$
Q^{\prime \prime}=Q^{\prime} \int_{0}^{t} v^{\prime \prime}(Q) Q^{\prime} d t^{\prime}=Q^{\prime} \int_{0}^{t}(v-m)(Q) Q^{\prime} d t^{\prime}<0
$$

whence

$$
\begin{aligned}
{\left[\int_{c}^{b} \sqrt{m_{0}}\right]^{4} } & \leq\left[\left(v^{\prime}-v\right)^{2}(Q) \text { at } x=c\right] \times 2\left[e^{Q(b)-Q(c)}-1\right] \\
& \leq\left[\left(v^{\prime}-v\right)^{2}(Q) \text { at } x=c\right] \times 2\left[e^{Q(c)-Q(a)}-1\right]
\end{aligned}
$$

and now the summability of the last line forces meas $(t \geq 0: v(Q) \leq 0$ at $x=b)$ to be finite; in particular, ${ }^{3} \int_{0}^{\infty} v_{-}(Q) d t>-\infty$ at $x=b$, by 6$)$. Obviously, there is nothing special about $x=b$ : the same is true at $x=a$ by a self-evident reprise. This was the goal of Step 2.

STEP 3 will confirm that $\int_{0}^{\infty} v_{+}(Q) d t$ is also finite at $x=a:\left(v^{\prime}-v\right)(Q)$ is positive between $x_{-}$and 0 and decreases there in view of $v^{\prime \prime}-v^{\prime}=-m+v-v^{\prime}<0$, so

$$
\exp \left[\int_{0}^{t} v^{\prime}(Q)-\int_{0}^{t} v(Q)\right]=Q^{\prime} e^{x-Q} \text { is } \geq 1 \text { and decreases, too. }
$$

Then

$$
\begin{aligned}
1 \leq Q^{\prime}(b) e^{b-Q(b)} & \leq \frac{1}{b-a} \int_{a}^{b} e^{x-Q(x)} d Q \\
& \leq \frac{e^{b}}{b-a} \times e^{-Q(a)}
\end{aligned}
$$

and now this estimate, controlling $Q(a)=a+\int_{0}^{t} v_{-}(Q)+\int_{0}^{t} v_{+}(Q)$ from above, confirms $\int_{0}^{\infty} v_{+}(Q) d t<\infty$.

STEP 4. But this means that $Q$ is bounded, not only if $x=a$, but at $x=c$ as well, by a self-evident reprise, and now $\left(\int_{c}^{b} \sqrt{m_{0}}\right)^{4}$ as seen in step 2 , is over-estimated

\footnotetext{
${ }^{3} v_{-}$is the negative part of $v$.

${ }^{4} v_{+}$is the positive part of $v$.
} 
by a constant multiple of $e^{Q}\left(v^{\prime}-v\right)^{2}(Q)$ at $x=c$, this being summable by 3 ) and the fact that $\left(v^{\prime}-v\right)(Q) \geq 0$. That is the contradiction.

Case 2: $\left(v^{\prime}-v\right)(Q) \geq 0$ at $x=0$ for all $t \geq 0$ and $\left(v^{\prime}+v\right)(Q)<0$ at $x=0$ for, e.g., $t=0$. Then 4) shows that, in the absence of breakdown, $\int_{0}^{\infty} e^{Q} d t<\infty$ at $x=0$ and so also for $x \leq 0, Q$ being increasing in $x$, in which case, $\int_{0}^{\infty} e^{-Q} d t=+\infty$ and $e^{Q}\left(v^{\prime}-v\right) \geq 0$ for every $x \leq 0$, by 3 ). Now $\int_{0}^{\infty} e^{Q} d t<\infty$ for $x \leq 0$ only if

$$
\infty>\int_{0}^{\infty} e^{Q} Q^{\prime} d t=\int_{0}^{\infty} \exp \left[\int_{0}^{t}\left(v^{\prime}+v\right)(Q) d t^{\prime}\right] d t \quad \text { a.e. }
$$

from which you learn that $\left(v^{\prime}-v\right)(Q)$ turns strictly negative, and stays that way, by 4), at sometime $T(x)<\infty$, for almost all $x \leq 0$; moreover, the fact that $e^{-Q}\left(v^{\prime}+v\right)(Q)$ decreases for $x_{-} \leq x \leq 0$ implies that $T(x)$ is finite and decreasing in the half-open interval $x_{-}<x \leq 0$. Two subcases are now distinguished according to the behavior of $\Delta Q=Q(b)-\bar{Q}(a)$ for $x_{-}<a<b<0$.

Case 2.1: $\Delta Q$ is bounded from above for every choice of $x_{-}<a<b<0$ (as it must be in the periodic case when $\Delta Q \leq Q(1)-Q(0)=1)$. Now $\infty>T(a)=0$, say, in which case $\left(v^{\prime}-v\right)(Q)>0,\left(v^{\prime}+v\right)(Q) \leq 0$, and $v(Q)<0$ for all $a \leq x \leq b$ and $t \geq 0$. Now comes a familiar type of trick:

$$
\begin{aligned}
{\left[\int_{a}^{b} \sqrt{m_{0}} d x\right]^{2}=\left[\int_{a}^{b} \sqrt{m} d Q\right]^{2} } & \leq \int_{a}^{b} m(Q) d Q \times \Delta Q \\
& \leq \int_{a}^{b}-v^{\prime \prime}(Q) d Q \times \Delta Q \\
& =-\left.v^{\prime}(Q)\right|_{a} ^{b} \times \Delta Q
\end{aligned}
$$

from which it appears that, in the absence of breakdown, $\Delta Q$ is also bounded from below, by 7) and 8). But also $Q^{\prime \prime}<0$ as in Step 2 of Case $1, v$ being negative, from which you learn that $0<Q^{\prime}(b) / Q^{\prime}(a)<1$ and that

$$
\frac{d}{d t} \frac{Q^{\prime}(b)}{Q^{\prime}(a)}=\left.v^{\prime}(Q)\right|_{a} ^{b} \times \frac{Q^{\prime}(b)}{Q^{\prime}(a)}<0 \text { is summable }
$$

$Q^{\prime \prime}<0$ and the boundedness of $\Delta Q$ for every choice of $x_{-}<a<b<0$ come into play once more to check that $Q^{\prime}(b) / Q^{\prime}(a)$ is bounded below, and now a contradiction is obtained: $\left.v^{\prime}(Q)\right|_{a} ^{b}$ is summable, but

$$
\left[\int_{a}^{b} \sqrt{m_{0}}\right]^{2} \leq\left. v^{\prime}(Q)\right|_{a} ^{b} \times \text { the upper bound of } \Delta Q \text { is not. }
$$

Case 2.2: $Q\left(b^{\prime}\right)-Q\left(a^{\prime}\right)$ is unbounded above for some $x_{-}<a^{\prime}<b^{\prime}<0$. Now pick $x_{-}<c<a<b<a^{\prime}$ with $T(c)=0$ for simplicity and $m_{0}(x)>0$ between $c$ and $b$. Note that $v(Q)$ is negative, as before, so that $v^{\prime \prime}(Q)<v(Q)<0, m(Q)$ being positive, and also that $Q^{\prime \prime}<0$. A variant of the familiar $\left[\int_{a}^{b} \sqrt{m_{0}} d x\right]^{2} \leq \int_{a}^{b} m(Q) d Q \times \Delta Q$ is 
now used. It reads

$$
\begin{aligned}
& {\left[\int_{a}^{b} \sqrt{m_{0}(x)} d x\right]^{2} \leq \int_{a}^{b} m(Q)[Q(x)-Q(c)] d Q \int_{a}^{b} \frac{d Q}{Q(x)-Q(c)}} \\
& <\int_{Q(c)}^{Q(b)}-v^{\prime \prime}(x)[x-Q(c)] d x \lg \frac{Q(b)-Q(a)}{Q(a)-Q(c)} \\
& \left.\begin{array}{l}
<-\left[v^{\prime}(Q) \text { at } x=b\right] \times Q(b)-Q(c) \\
\quad-[v(Q) \text { at } x=c]
\end{array}\right\} \times \ell g \frac{b-a}{a-c},
\end{aligned}
$$

$Q^{\prime \prime}<0$ being used to appraise the logarithm. I propose to show that $v^{\prime}(Q)>0$ at $x=b$ and that $v(Q)=o(1)$ at $x=c$ for suitable $t=t_{1}<t_{2}<$ etc. $\uparrow \infty$. This will be contradictory. Chose these "special" times so that $Q\left(b^{\prime}\right)-Q\left(a^{\prime}\right) \uparrow+\infty$. Then $Q\left(a^{\prime}\right)-$ $Q(b) \uparrow+\infty$, too, since $Q^{\prime \prime}<0$, and you can also assume $(d / d t)\left[Q\left(a^{\prime}\right)-Q(b)\right]>0$ at special times. But

$$
\frac{d}{d t}\left[Q\left(a^{\prime}\right)-Q(b)\right]=\int_{b}^{a^{\prime}} v^{\prime}(Q) d Q \leq\left[v^{\prime}(Q) \text { at } x=b\right] \times\left[Q\left(a^{\prime}\right)-Q(b)\right]
$$

so $v^{\prime}(Q)$ is positive at $x=b$ for special times and stays positive down to $x_{-}$in view of $v^{\prime \prime}(Q)<0$. This is half the battle. Next, fix $d<c$. Then $-v(Q)$ decreases between $d$ and $c$, so

$$
0<-[v(Q) \text { at } x=c] \times[Q(c)-Q(d)]<-\int_{d}^{c} v^{\prime \prime}(Q) d Q,
$$

in which the right hand side is bounded in the absence of breaking, and since $Q(c)$ $Q(d) \uparrow+\infty$ by reason of $Q^{\prime \prime}<0$, you may conclude that $v(Q)=o(1)$ at $x=c$ for special times. The proof is finished.

4. How It Breaks Down. The conditions for breaking are now established, but how does it happen?

Return to fig. 1 with $m(Q)>0$ for $x_{-}<x<a, m(Q)=0$ for $a \leq x \leq b$, and $m(Q)<0$ for $b<x<x_{+}$; necessarily, $a \leq 0 \leq b . T<\infty$ is the breaking time, i.e., the moment when $v^{\prime}(Q)$ gets out of hand, assuming that this takes place between $x_{-}$ and $x_{+}$. Breakdown can happen only in this way, as will be seen.

Item 1. $v^{\prime}(Q) \equiv w$ is bounded above, by 7), and 5) in the form $m^{\bullet} \leq \frac{1}{2} C^{2}-\frac{1}{2} w^{2}$ shows that it can get out of hand only by an ultimate decrease to $-\infty$, in the style of $w=$ a negative constant $\times(T-t)^{-1}$ or worse, in which case $Q^{\prime}(t, x)=\exp \int_{0}^{t} v^{\prime}(Q) \downarrow$ 0 as $t \uparrow T$, and conversely: if $Q^{\prime} \downarrow 0$ as $t \uparrow T<\infty$, then $w=v^{\prime}(Q)$ is ultimately very negative and 5) drives it down to $-\infty$.

Item 2. $Q(T-, x)$ exists everywhere, $v$ being bounded, and $e^{Q}\left(v^{\prime}-v\right)(Q)$ decreases/increases where $m(Q)$ is positive/negative, from which you see that breakdown occurs, if at all, at points where $m(Q)$ changes sign, from positive to negative: $x=0$ is such a point.

Item 3. $v^{\prime}(Q)$ at $x=0$ now decreases to $-\infty$ as $t \uparrow T$ and carries with it the slope $v^{\prime}(Q)$ at the general point $x$ of the (maximal) interval $[a, b]$ where $m(Q)=0$, $v^{\prime \prime}=v$ being bounded there; in particular, $Q(x)$ flattens out in the whole interval $a \leq x \leq b$, by item 1 .

Item 4 is the converse: if $Q(x)$ flattens out in any interval $a \leq x \leq b$, then $m_{0}(x)$ and so also $m(Q)$ vanishes there; simultaneously, $v^{\prime}(Q) \downarrow-\infty$. 
Proof. Let $Q(x)$ flatten out in $a \leq x \leq b$ with $x_{-}<a<b \leq 0$, say, and take $c<d$ properly between $a$ and $b$. Then

$$
\begin{aligned}
& {\left[\int_{c}^{d} \sqrt{m_{0}} d x\right]^{2} \leq \int_{c}^{d} m(Q)[Q(b)-Q(x)] d Q \int_{c}^{d} \frac{d Q}{Q(b)-Q(x)}} \\
& \leq \int_{c}^{b}\left(v-v^{\prime \prime}\right)(Q)[Q(b)-Q(x)] d Q \times \ell g \frac{Q(b)-Q(c)}{Q(b)=Q(d)} \\
& \leq C \times \frac{1}{2}[Q(b)-Q(a)]^{2} \\
& \left.\begin{array}{l}
+v^{\prime}(Q) \text { at } x=a \times[Q(b)-Q(a)] \\
-\left.\quad v(Q)\right|_{a} ^{b}
\end{array}\right\} \times \ell g \frac{Q(b)-Q(c)}{Q(b)-Q(d)} \\
& \leq \text { a constant multiple of } \sqrt{Q(b)-Q(a)} \times \ell g \frac{Q(b)-Q(a)}{Q(b)-Q(d)}
\end{aligned}
$$

owing to the control of $v^{\prime}(Q)$ above, by 7), and to the elementary estimate $\mid v(b)-$ $\left.v(a)\right|^{2} \leq \int_{a}^{b}\left(v^{\prime}\right)^{2} \times(b-a)$, the integral being controlled by the proper constant of motion in all function classes. Now $Q^{\prime \prime}=Q^{\prime} \int_{0}^{t} v^{\prime \prime}(Q) Q^{\prime}$ is bounded above by a multiple $K$ of $Q^{\prime}$ for $a \leq x \leq b$ in view of $m=v-v^{\prime \prime}>0$ and 7); in particular, $Q^{\prime} e^{-K Q}$ is decreasing in $x$, so the flattening out of $Q$ in $[a, b]$ implies that, for $t \uparrow T$, $Q^{\prime} \downarrow 0$ and so also $v^{\prime}(Q) \downarrow-\infty$, uniformly in $[a, b]$.

Now use 5) for $w=v^{\prime}(Q)$ in the form $w^{\bullet}=-\frac{1}{2} w^{2}+2 V$, with $V=\frac{1}{4} v^{2}(Q)$ etc., and write $w=2 \psi^{\bullet} / \psi$ with $\psi(0)=1$, say, and $\psi^{\bullet}(0)<0$, fixing the variable $x$ between $a$ and $b$. Then $\psi^{\bullet \bullet}=V \psi, \psi(T-)=0$, and $\psi^{\bullet}(T-)<0^{5}$, reflecting the fact that $w \downarrow-\infty$ as $t \uparrow T$. It follows that $v^{\prime}(Q)$ behaves like $-2 /(T-t)$ or nearly so as $t \uparrow T$, so $Q^{\prime}$ is no worse than $(T-t)^{3 / 2}$ for $a \leq x \leq b$. But now $\int_{c}^{d} \sqrt{m_{0}}$, as seen in the big display, is over-estimated by $(T-t)^{3 / 4} \ell g(T-t)$ and so vanishes, and $m_{0}(x)=0$ in whole of $[a, b]$, the subinterval $[c, d]$ having been chosen as you will.

5. A Little Example. This falls outside the function classes admitted above but never mind. $v(t, x)$ is soliton/anti-soliton pair

$$
\frac{1}{2} p e^{-|x+q|}-\frac{1}{2} p e^{-|x-q|}
$$

symmetric about $x=0$, with positive $q=q(t)$ and $p=p(t), q^{\bullet}=-p\left(1-e^{-2 q}\right)$, $p^{\bullet}=p^{2} e^{-2 q}$, and the constant of motion $H=\frac{1}{2} p^{2}\left(1-e^{-2 q}\right)$. With $\Theta=T-t$, you find $q=\lg \operatorname{ch} \Theta, p=\operatorname{ch} \Theta / \operatorname{sh} \Theta$ and, for $x>\lg \operatorname{ch} T=q(0), e^{Q}=e^{x}+\operatorname{ch} \Theta-\operatorname{ch} T$, as you may check. In particular, $Q$ flattens out and $v^{\prime}(Q) \downarrow-\infty$ in the interval $|x| \leq q(0)=\lg \operatorname{ch} T$, precisely.

\footnotetext{
${ }^{5} \psi^{\bullet}(T-)=0$ implies $\psi \equiv 0$ which is not the case.
} 


\section{REFERENCES}

[1] V. I. Arnold And B. A. Khesin, Topological Methods in Hydrodynamics, Appl. Math. Sci., 125, Springer-Verlag, New York, 1998.

[2] R. CAmassa AND D. D. Holm, An integrable shallow water equation with peaked solitons, Phys. Rev. Lett., 71 (1993), pp. 1661-1664.

[3] R. Camassa, D. D. Holm, And J. Hyman, A new integrable shallow water equation, Adv. Appl. Math., 31 (1994), pp. 1-33.

[4] A. ConstANTIN AND J. EScher, Well-posedness, global existence, and blow-up phenomena for a periodic quasi-linear hyperbolic equation, CPAM, 51 (1998), pp. 475-504.

[5] A. Constantin And H. P. MCKean, A shallow-water equation on the circle, CPAM, to appear 1999 .

[6] A. FoKAs AND B. FuchsteIner, Symplectic structures, their Bäcklund transformations, and hereditary symmetries, Physica D., 4 (1981), pp. 47-66.

[7] G. Misiolek , A shallow water equation as geodesic flow on the Bott-Virasoro group, J. Geom. Phys., 24 (1998), pp. 203-208. 